\title{
Synthesis, characterisation and application in coagulation experiments of polyferric sulphate
}

\author{
A. Zouboulis, V. Fotini \& M. Panagiotis \\ Division of Chemical Technology, Department of Chemistry, \\ Aristotle University of Thessaloniki, Greece
}

\begin{abstract}
The process of coagulation is a core environmental protection technology, which is mainly used in the wastewater treatment facilities. Research is now focused on the development of new inorganic coagulants. A characteristic example is PFS (polyferric sulphate), a relatively new pre-polymerised inorganic coagulant with high cationic charge. It consists of medium and high molecular weight polymeric chains and it can be described with the chemical formula $\left[\mathrm{Fe}_{2}(\mathrm{OH})_{\mathrm{n}}\left(\mathrm{SO}_{4}\right)_{(6-\mathrm{n}) / 2}\right]_{\mathrm{m}}$. In this paper, the role of some parameters including temperature, types of chemical reagents etc in the preparation stages of PFS were investigated. Furthermore, the prepared PFS was characterized based on typical properties such as the percentage of the polymerised iron present in the compound etc. Furthermore, dynamics of coagulation process were examined by means of the Photometric Dispersion Analyzer (PDA). Finally, the coagulation performance of PFS in reducing the turbidity of synthetic kaolinite suspensions was studied.

Keywords: coagulation, polyferric sulphate, inorganic polymeric coagulant, prepolymerised coagulant.
\end{abstract}

\section{Introduction}

The process of coagulation is widely used in the wastewater treatment facilities especially for the destabilisation of colloids suspensions and for the removal of suspended solids along with the removal of phosphate ions. It is known as a core environmental protection technology. Nearly all the colloids found in natural waters carry negative charge and therefore, they remain in suspension, due to the mutual electric repulsions. Therefore, the addition of a cation will result in colloidal destabilisation, as they specifically interact with the negatively charged colloids and neutralise their charge. Highly charged cation, such as $\mathrm{Fe}^{3+}$, is 
regarded as one of the most effective cation for such a purpose. Therefore, there are a number of conventional coagulants based on iron such as the $\mathrm{Fe}_{2}\left(\mathrm{SO}_{4}\right)_{3}$ and the $\mathrm{FeCl}_{3}$. However, current research focuses on the development of new type of coagulants, which will combine superior efficiency and lower operational cost as compared with the conventional coagulants.

A relatively new inorganic coagulant is the polyferric sulphate (PFS). PFS is a pre-hydrolysed coagulant, which can be described by the chemical formula $\left[\mathrm{Fe}_{2}(\mathrm{OH})_{\mathrm{n}}\left(\mathrm{SO}_{4}\right)_{(6-\mathrm{n}) / 2}\right]_{\mathrm{m}}$, where $\mathrm{n}<2$ and $\mathrm{m}>10$. It contains polynuclear complex ions, such as $\mathrm{Fe}_{2}(\mathrm{OH})_{2}{ }^{4+}, \mathrm{Fe}_{3}(\mathrm{OH})_{4}{ }^{5+}$, formed by $\mathrm{OH}$ bridges and a large quantity of inorganic macromolecular compounds. The molecular weight can be as high as $10^{5}$ (Chang and Wang [1]). Due to the presence of polymeric species the PFS carries high cationic charge, which can improve the charge neutralising capacity and hence, it becomes more effective at a comparatively lower dose, than the conventional coagulants. (Jiang and Graham [2]). Furthermore, it has been reported by several researchers (Chang and Wang [1], Jiang and Graham [2], Jiang and Graham [3], Butler et al. [4], Li et al. [5]), that PFS exhibits a superior efficiency in the removal of chemical oxygen demand (COD), biochemical oxygen demand (BOD), turbidity and colour, than the conventional coagulants based on iron. Also, it can be used in a wide range of $\mathrm{pH}$ and temperature, due to its strong hydrolysis. Meanwhile, some preliminary toxicity studies suggested that drinking water treated with PFS is safe for consumption (Hendrich et al. [6]).

Several researchers have proposed different methods for preparing the PFS in laboratory scale (Jiang and Graham [7], Fan et al. [8], Cheng [9]). However, the role of certain parameters, such as the temperature, the type of chemical reagents in the various stages of preparation methods has not been thoroughly examined. Therefore, the aim of this paper is the investigation of the optimum experimental conditions for the synthesis of PFS. Furthermore, the coagulation performance of PFS is assessed by examining the efficiency in removing the turbidity of simulated kaolinite water suspensions. The specific conditions applied in the coagulation experiments were found by means of the Photometric Dispersion Analyser (PDA).

\section{Experimental procedures}

\subsection{Mechanism of PFS preparation}

The synthesis of PFS commences with the oxidation of ferrous sulphate $\left(5.59 \times 10^{-3} \mathrm{M}\right.$ as $\left.\mathrm{Fe}\right)$ to ferric sulphate in highly acidic conditions $\left(\mathrm{H}_{2} \mathrm{SO}_{4}\right.$, $96 \mathrm{wt} \%)$. The oxidising agent was nitric acid $\left(\mathrm{HNO}_{3}, 65 \mathrm{wt} \%\right)$.

$$
\mathrm{FeSO}_{4}+1 / 2 \mathrm{SO}_{4}{ }^{2-}+\text { oxidising agent } \rightarrow 1 / 2 \mathrm{Fe}_{2}\left(\mathrm{SO}_{4}\right)_{3}
$$

When the amount of sulfuric acid is limited, the hydroxide ion will replace the sulphate ion in the hydrolysis stage and therefore, the polymerisation will occur:

$$
\begin{aligned}
& \mathrm{Fe}_{2}\left(\mathrm{SO}_{4}\right)_{3}+\mathrm{nOH}^{-} \rightarrow \mathrm{Fe}_{2}(\mathrm{OH})_{\mathrm{n}}\left(\mathrm{SO}_{4}\right)_{3-\mathrm{n}}+\mathrm{n} \mathrm{SO}_{4}{ }^{2-} \text { (Hydrolysis) } \\
& \mathrm{mFe}_{2}(\mathrm{OH})_{\mathrm{n}}\left(\mathrm{SO}_{4}\right)_{3-\mathrm{n} / 2} \rightarrow\left[\mathrm{Fe}_{2}(\mathrm{OH})_{\mathrm{n}}\left(\mathrm{SO}_{4}\right)_{3-\mathrm{n} / 2}\right]_{\mathrm{m}} \text { (Polymerisation) }
\end{aligned}
$$




\subsection{Synthesis of PFS}

The synthesis of the PFS was performed following the proposed method of Jiang and Graham [7]. The investigation of optimum experimental conditions was conducted by altering certain experimental parameters, such as the temperature of each stage of the preparation method, as well as the type and the amount of chemical reagents used.

\subsection{Characterisation of the PFS}

\subsubsection{Determination of total iron concentration}

The total iron concentration was measured by means of Atomic Absorption Spectroscopy (Perkin-Elmer 2380) (Clesceri et al. [10]).

\subsubsection{Determination of polymerised iron concentration}

The measurement of the Fe species by the ferron-timed spectroscopy method has been reported previously (Jiang and Graham [7]). The ferron reagent (8-hydroxy7-iodoquinoline-5-sulphonic acid) can form complexes with single ferric ion, monomeric and dimeric species within the reaction time of $1 \mathrm{~min}$, while it can complex with medium and high molecular weight polymers with increasing reaction time. Precipitated ferric species will not react with ferron. Based on this principle, visible absorbance at $600 \mathrm{~nm}$ was measured as a function of time and each absorbance corresponded to the respective ferric concentration.

\subsubsection{Determination of ferrous ion concentration}

A potassium permanganate titration method was used to determine the ferrous ion concentration in the PFS. The method can be described by the following equation:

$$
\mathrm{MnO}_{4}^{-}+5 \mathrm{Fe}^{2+}+8 \mathrm{H}^{+} \rightarrow \mathrm{Mn}^{2+}+5 \mathrm{Fe}^{3+}+4 \mathrm{H}_{2} \mathrm{O}
$$

\subsubsection{Determination of the ratio $\mathrm{r}=[\mathrm{OH}-] /\left[\mathrm{Fe}^{3+}\right]$}

The molar ratio of $\mathrm{OH}^{-}$to the $\mathrm{Fe}^{3+}$ is called the $\mathrm{r}$ value and it can be directly related to the stability of the PFS. In particular, for high values of $r$, e.g. $r>0,4$, the prepared PFS is unstable and precipitates may emerge. It is determined by adding excess hydrochloric acid $(\mathrm{HCl} 1 \mathrm{~N})$ and potassium fluoride solution (KF $50 \%$ wt to a sample) and back titrating with sodium hydroxide $(\mathrm{NaOH} 1 \mathrm{~N})$.

\subsection{Photometric Dispersion Analyser (PDA)}

The extent of aggregation, as well as the kinetics of coagulation was examined with the aid of Photometric Dispersion Analyser (PDA 2000, Rank Brothers Bottisham UK). The PDA instrument measures the ratio R, or the flocculation index FI, which are directly related with the mean concentration and size of dispersed particles. The test suspension $(1.5 \mathrm{~L})$ was contained into a 2 - $\mathrm{L}$ beaker and was constantly stirred by a JP SELECTA jar test device. The created microflocs passed through the measuring transparent plastic cuvette $(3 \mathrm{~mm}$ diameter) with the aid of a peristaltic pump. The applied flow rate was $30 \mathrm{ml} / \mathrm{min}$ 
in order to secure laminar conditions throughout the experiment. The ratio $\mathrm{R}$ curves derived from the PDA instrument have the typical form shown in Figure 1 (Hopkins and Ducoste [11]).

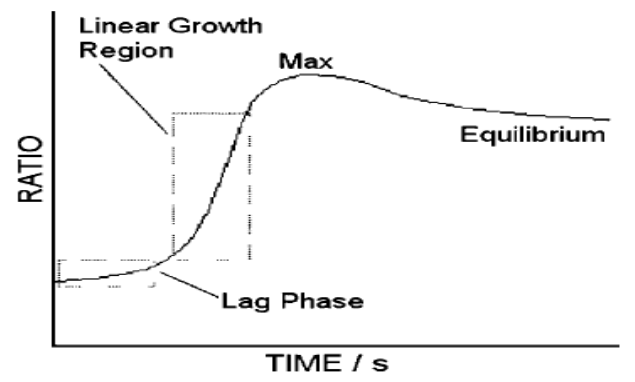

Figure 1: $\quad$ Schematic representation of the typical ratio $\mathrm{R}$ curve.

The $\mathrm{R}$ curve can be divided into three parts: A) immediately after the coagulant addition there is a small change in the $\mathrm{R}$ values. During this part, which is called "lag phase", the destabilisation of the particles takes place. B) Then, the linear growth region part follows, where the collisions of previously destabilized particles result in aggregate formation. C) Finally, in the equilibrium phase the value of the $\mathrm{R}$ remains relatively constant as the rate of the formation and the breakage of flocs is rather equal. Generally, it can be concluded that the higher $\mathrm{R}$ values imply bigger particles size and therefore, a better separation by the application of subsequent sedimentation.

\subsection{Jar test}

The coagulation experiments were carried out using a jar test apparatus with six paddles (Aqualytic). Kaolinite water suspension was used as the test suspension, dosed with the appropriate amount of the coagulant. The conditions, under which the experiments were conducted, were determined from the study of coagulation dynamics in accordance with the relevant literature (Fan et al. [8], Jiang and Graham [2]). In particular, the fast mixing time was set to $3 \mathrm{~min}$ at a paddle speed of $300 \mathrm{rpm}$ to allow the particles to be destabilized. The flocculation period was $30 \mathrm{~min}$ at a paddle speed of $35 \mathrm{rpm}$ and the sedimentation period lasts for $45 \mathrm{~min}$. After that period a supernatant sample $(50 \mathrm{ml})$ was withdrawn for turbidity measurements. Turbidity measurements were carried out by using a HACH RATIO/XR Turbimeter.

\section{Results and discussion}

\subsection{Characterisation of PFS}

Table 1 shows the characteristics of the PFS produced, as well as the conditions under which their synthesis occurred. The use of weak base solution in contrast 
Waste Management and the Environment III 137

\begin{tabular}{|c|c|c|c|c|c|c|}
\hline$\underbrace{+\infty}$ & ֻุ & $\frac{1}{3}$ & $\frac{0}{0}$ & ָุ & ָุ & $\stackrel{+}{\circ}$ \\
\hline$\underset{2}{Z}$ & $\stackrel{n}{\sim}$ & $\exists$ & $\stackrel{\simeq}{-}$ & $\stackrel{ナ}{\sim}$ & $\stackrel{n}{-}$ & $N$ \\
\hline 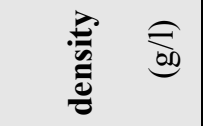 & $\stackrel{N}{=}$ & $\bar{\Xi}$ & $\stackrel{\infty}{=}$ & $\stackrel{\infty}{=}$ & $\stackrel{0}{\varrho}$ & $\underset{\sigma}{\sigma}$ \\
\hline 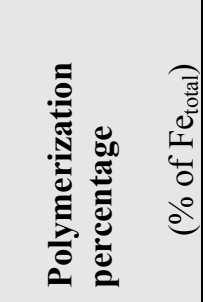 & $\stackrel{\infty}{m}$ & $\beth$ & $\stackrel{\bullet}{\sim}$ & 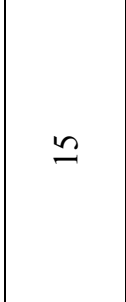 & 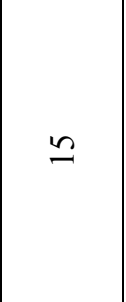 & $=$ \\
\hline : & ñ & ñ & $\stackrel{n}{n}$ & ñ & $\stackrel{n}{n}$ & $\stackrel{n}{\sim}$ \\
\hline 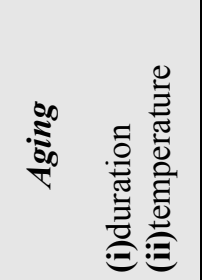 & 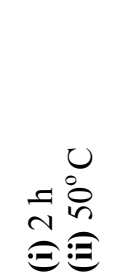 & 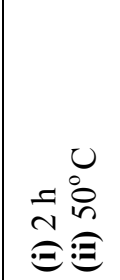 & 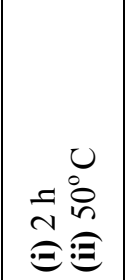 & 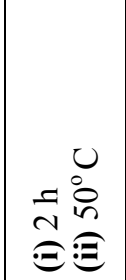 & 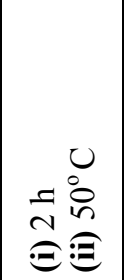 & 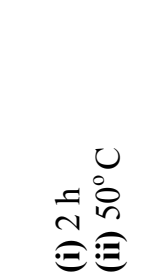 \\
\hline 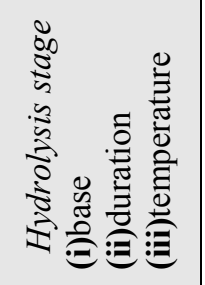 & 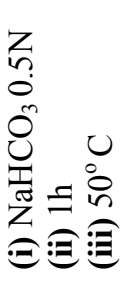 & 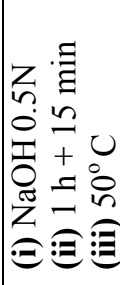 & 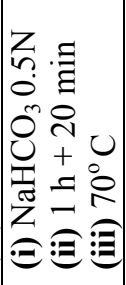 & 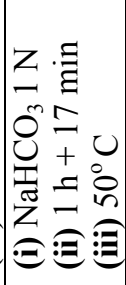 & 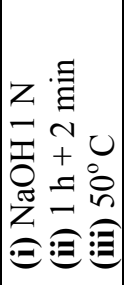 & 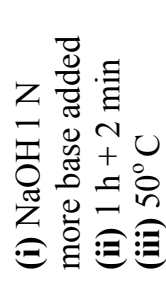 \\
\hline 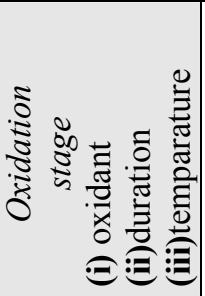 & 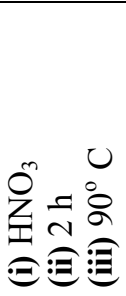 & 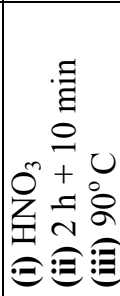 & 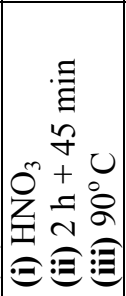 & 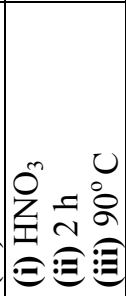 & 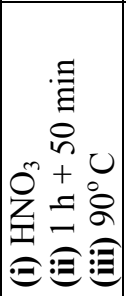 & 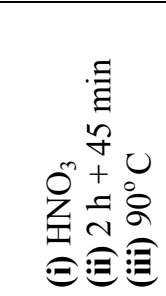 \\
\hline & $\ll$ & $\oplus$ & U & ค & 되 & {$[x$} \\
\hline
\end{tabular}


with the use of a strong base results in the increase of polymerisation percentage, of the ratio $\mathrm{r}=\left[\mathrm{OH}^{-}\right] /\left[\mathrm{Fe}^{3+}\right]$ and of the product stability during the following 4month period (precipitate appearance). Additionally, Table 2 shows a comparison between the PFS produced in the laboratory and those produced according to the relevant literature.

Table 2: $\quad$ Comparative table of the PFS produced in the laboratory and those produced according to the relevant literature.

\begin{tabular}{|c|c|c|c|c|c|c|}
\hline & $\begin{array}{l}\mathbf{F e}_{\text {total }} \\
(\mathbf{g} / \mathbf{l})\end{array}$ & $\begin{array}{l}\text { Polymerized Fe } \\
\left(\% \text { of } \mathbf{F e}_{\text {total }}\right)\end{array}$ & $\mathbf{p H}$ & $\begin{array}{l}{\left[\mathbf{O H}^{-}\right.} \\
\mathbf{J / [ F ^ { + 3 } ]}\end{array}$ & $\begin{array}{l}\text { density } \\
(\mathbf{g} / \mathbf{l})\end{array}$ & $\begin{array}{l}\% \\
\text { turbidity } \\
\text { removal }\end{array}$ \\
\hline $\begin{array}{c}\text { laboratory } \\
\text { PFS }\end{array}$ & 52.5 & 38 & 1.5 & 0.25 & 1182 & $95^{*}$ \\
\hline $\begin{array}{c}\text { Jiang and } \\
\text { Graham [7] }\end{array}$ & 40 & 65.2 & 1 & 0.3 & - & $96^{* *}$ \\
\hline Cheng [12] & 150 & - & 0.56 & 0.4 & 1480 & - \\
\hline
\end{tabular}

* kaolinite suspension, ${ }^{* *}$ surface water with algae

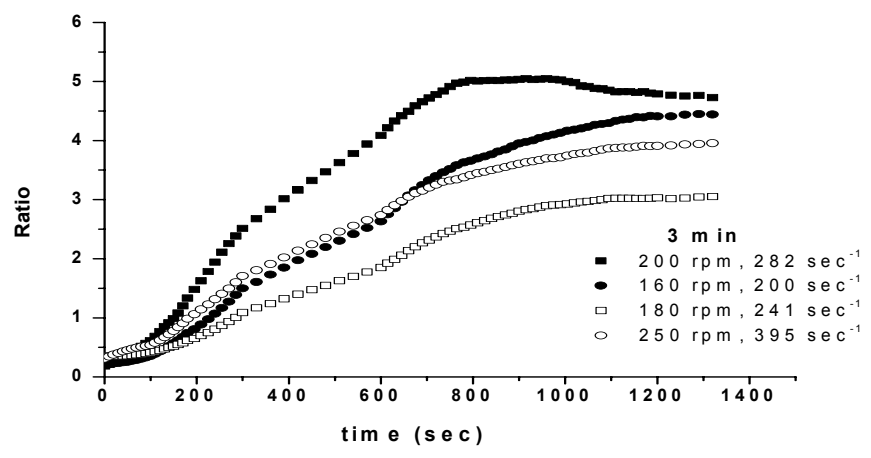

Figure 2: Impact of fast mixing speed (or velocity gradient $\mathrm{G}\left(\mathrm{s}^{-1}\right)$ ) on the coagulation process of kaolinite water suspension $(5 \mathrm{mg} / \mathrm{L})$; $\operatorname{PFS}(5 \mathrm{mg} / \mathrm{l}), \mathrm{pH}=7$.

\subsection{Study of kinetics of the coagulation using the PDA}

\subsubsection{Effect of mixing speed (or velocity gradient $G\left(s^{-1}\right)$ ) in the kinetics of coagulation}

The effect of speed during the fast mixing period on the coagulation process is shown in Figure 2. The mixing speed is expressed in rpm, as well as in velocity gradient units $\mathrm{G}\left(\mathrm{s}^{-1}\right)$. As shown in Fig. 2, for the mixing speed of $200 \mathrm{rpm}$, the "lag" phase is limited and this results in a faster flocculation. Also, for the same speed a relatively higher speed of flocculation is achieved and the final flocs after the equilibrium of the system have a larger size. In the contrary, for the mixing speed of $160 \mathrm{rpm}$, the "lag" phase has a bigger duration and therefore the formation of flocs is delayed. The flocculation of suspended particles proceeds in 
lower speed and the final size of flocs is smaller. The same happens in the case of very high mixing speed $(250 \mathrm{rpm})$. The small size of flocs is due to the fact that the smaller flocs, which are formed during the fast mixing period, are destroyed by the shear stress which develops during that stage and therefore, it is impossible to obtain a bigger size in the flocculation stage.

\subsubsection{Effect of mixing time in the kinetics of coagulation process}

Figure 3 shows the effect of mixing time in the kinetics of coagulation process. The speed in the fast mixing stage is $200 \mathrm{rpm}$, which found to be the optimum as shown in the previous section 3.3.1. The higher values of ratio, which correspond to the bigger size of formed flocs, were achieved, when the fast mixing time was $180 \mathrm{~s}$. It is worth noting that the "lag" phase in both cases is similar and the fact which distinguishes the two curves, is the bigger size of the flocs in the slow mixing period.

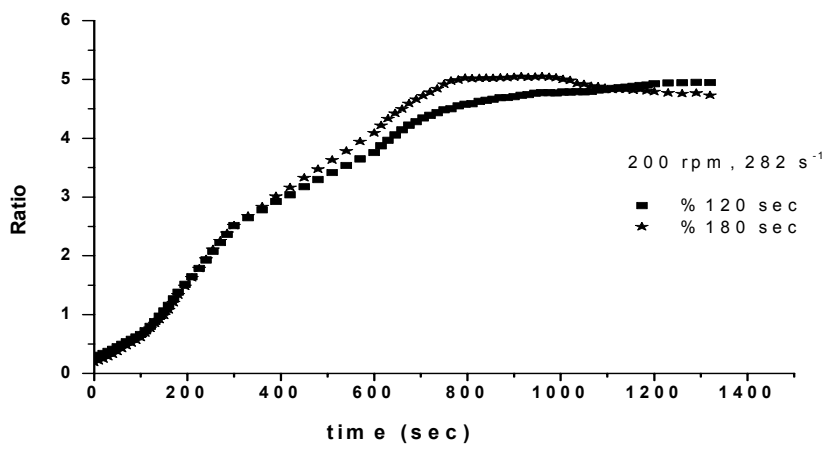

Figure 3: Impact of time during the fast mixing period in kaolinite water suspension ( $5 \mathrm{mg} / \mathrm{L})$; PFS (5mg/l), $\mathrm{pH}=7$.

\subsection{Jar test results}

\subsubsection{Comparative jar test results among the prepared PFS}

Figure 4 summarises the comparative results among the different PFS. The test solution was kaolinite water suspension $(5 \mathrm{mg} / \mathrm{L})$, with initial turbidity 8 NTU and $\mathrm{pH}=7$. The polyelectrolyte used was the non-ionic Magnafloc LT20, for a dosage corresponding to the $1 / 10$ th of the coagulant dosage. The suspensions were stirred rapidly in $200 \mathrm{rpm}$ for $3 \mathrm{~min}$, the flocculation period was $30 \mathrm{~min}$ (in $35 \mathrm{rpm}$ ) and finally the flocs were left to settle for $45 \mathrm{~min}$. Figure 4 shows that higher turbidity removal was achieved when using PFS A, which is directly related to the higher degree of polymerization of that coagulant. PFS A and C, which are synthesized using $\mathrm{NaHCO}_{3}(0.5 \mathrm{~N})$ solution, showed better performance, than those produced with $\mathrm{NaOH}(0.5 \mathrm{~N})(\mathrm{B}, \mathrm{E}, \mathrm{F})$.

In general, the polymeric species carry high cationic charge, because of their larger size, which results in increasing surface activity and thus, improving their ability to neutralize the charge of suspended particles. Therefore, the increase in 
the polymerization percentage results in the increase of coagulating performance (Jiang and Graham [7]).

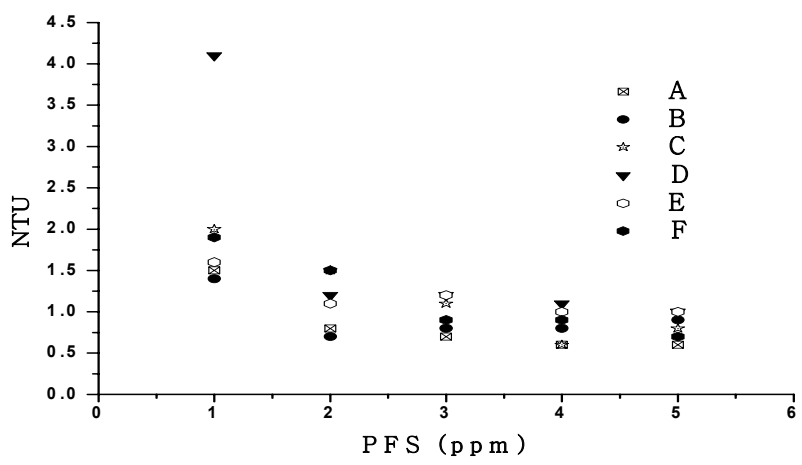

Figure 4: Turbidity removal of kaolinite water suspension $(5 \mathrm{mg} / \mathrm{L})$ against the PFS dosage, for polyelectrolyte addition equal to $1 / 10$ th of the coagulant dosage.

\subsubsection{Correlation between the degree of polymerisation and the removal of turbidity}

From the conducted experiments it was shown that an increase in the polymerization percentage could enhance the ability of the PFS to remove turbidity, which is confirmed by the relevant literature as well (Jiang and Graham [7]). The above statement is shown in Figure 5.

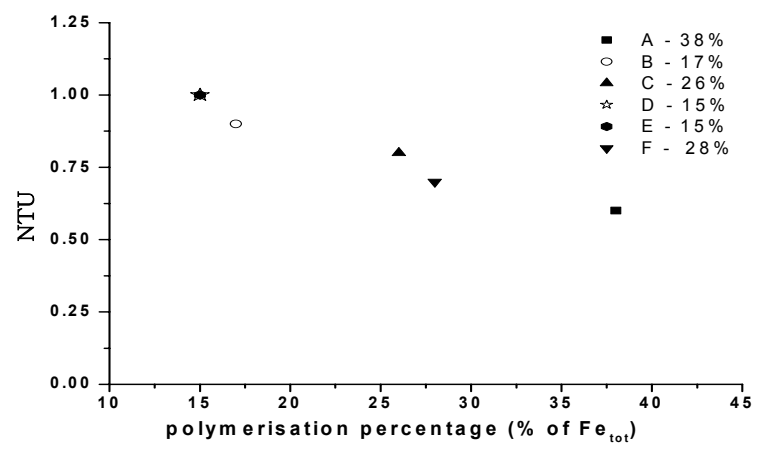

Figure 5: Turbidity removal against the polymerisation percentage, for a coagulant dose of $5 \mathrm{mg} / \mathrm{L}$ and polyelectrolyte dose $0.5 \mathrm{mg} / \mathrm{L}$.

\subsubsection{Comparative results between PFS and FS}

Furthermore, jar tests were conducted using the FS in order to compare its performance with the PFS produced in the laboratory. The ferric sulphate (FS) is 
a well-established conventional coagulant with a wide range of applications. The jar test conditions were similar with those used in previous experiments, with polyelectrolyte Magnafloc LT20 using a dosage of 1/10th of the coagulant dosage. The results are presented in Figure 6.

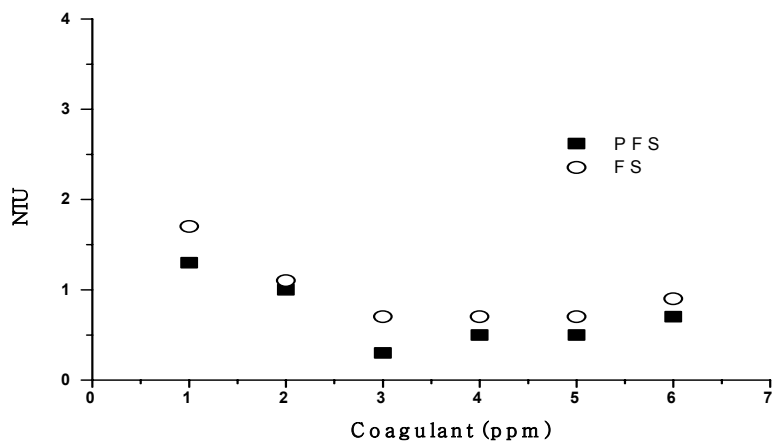

Figure 6: Turbidity removal of a kaolinite water suspension ( $5 \mathrm{mg} / \mathrm{l})$ against the two different coagulants: PFS and FS.

Figure 6 shows that the PFS exhibits a better performance in removing turbidity, than the FS. Although, a similar number of flocs were formed during the flocculation period of the jar test, the flocs of PFS were bigger in size and inevitably easier to settle. This is due to the fact that the polymeric species of PFS have the ability to form larger and therefore heavier flocs, which are easier to settle.

\section{Conclusions}

The use of a weak base $\left(\mathrm{NaHCO}_{3}\right)$ as a reducing agent results in the increase of polymerisation degree of PFS, of the ratio $r$, as well as in improving the stability of the product. Furthermore, the coagulating performance of prepared coagulants is enhanced.

High temperature $\left(90^{\circ} \mathrm{C}\right)$ in the oxidation stage and intermediate temperature $\left(50^{\circ} \mathrm{C}\right)$ in the hydrolysis and polymerisation stage results in increasing the degree of polymerisation along with its coagulating efficiency.

The presence of polymeric species is directly related to the coagulating efficiency of the PFS. In particular, the higher the polymerisation percentage the better the performance in removing the turbidity from kaolinite water suspensions.

PFS exhibits a higher coagulating efficiency compared with a conventional coagulant, e.g. $\mathrm{Fe}_{2}\left(\mathrm{SO}_{4}\right)_{3}$ under the specific experimental conditions. 


\section{Acknowledgments}

Thanks are due to the Greek Ministry of Education for funding this research through the Pythagoras II program.

\section{References}

[1] Chang Q., Wang H., "Preparation of PFS coagulant by sectionalised reactor, Journal of Environmental Sciences, 14(3),345-350, 2002

[2] Jiang J-Q., Graham N.J.D., "Coagulation of upland coloured water with polyferric sulphate compared to conventional coagulants", J. Water SRTAqua, 45(3), 143-154, 1996

[3] Jiang J-Q. and Graham N.J.D.,'Observations of the comparative hydrolysis/precipitation behavior of polyferric sulphate and ferric sulphate", Water Res., 32 (3), 930-935, 1998

[4] Butler A.D., Fan M., Brown R.C., Cooper A.T., van Leeuwen J.H., Sung S., " Absorption of dilute $\mathrm{SO} 2$ gas stream with conversion to polymeric ferric sulphate for use in water treatment", Chemical Engineering Journal, 98, 265-273, 2004

[5] Li F., Ji G., Gi X., “ The preparation of inorganic coagulant - Polyferric sulphate”, J. Chem. Tech. Biotechnol.”, 68, 219-221, 1997.

[6] Hendrich S., Fan M., Sung S., Brown R.C., Semakaleng R., Myers G., "Toxicity evaluation of polymeric ferric sulphate", Int J. Environ. Technol. Managem., 1, 464-471, 2001

[7] Jiang J-Q., Graham N.J.D., "Preparation and Characterisation of an optimal polyferric sulphate (PFS) as a coagulant for water treatment.", J.Chem.Technol.Biotechnol., 73, 351-358, 1998

[8] Fan M., Sung S., Brown R.C., Wheelock T.D. and Laabs F.C., "Synthesis, characterization and coagulation of polymeric ferric sulphate", J. Env. Engineering, 128 (6), 483-490, 2002

[9] Cheng W.P., "Comparison of hydrolysis/coagulation behavior of polymeric and monomeric iron coagulants in humic acid solution", Chemosphere, 47, 963-969, 2002

[10] Clesceri L., Greenberg A. and Trussell R., "Standard methods for the examination of water and wastewater", $17^{\text {th }}$ Ed., APHA-AWWA-WEF, Washington DC, 1989.

[11] Hopkins D. and Ducoste J., "Characterising flocculation under heterogeneous turbulence”, J. Coll. Interf. Sci., 264, 184-194, 2003

[12] Cheng W.P., "Hydrolysis characteristics of polyferric sulfate coagulant and its optimal condition of preparation", Colloids and Surfaces, 182, 57-63, 2001. 